\title{
Pathogenesis of chronic constipation in a Polish group of paediatric patients - an attempt to create the optimal histopathological diagnostic protocol
}

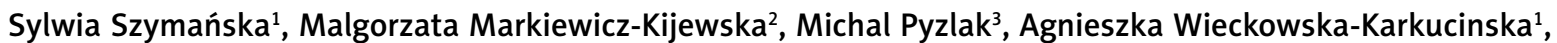 \\ Piotr Kalicinski ${ }^{2}$, Wiesława Grajkowska ${ }^{1}$, Maciej Pronicki ${ }^{1}$ \\ ${ }^{1}$ Department of Pathology, The Children's Memorial Health Institute, Warsaw, Poland \\ ${ }^{2}$ Department of Surgery and Organ Transplantation, The Children's Memorial Health Institute, Warsaw, Poland \\ ${ }^{3}$ Department of General and Experimental Pathology, Medical University of Warsaw, Warsaw, Poland
}

Gastroenterology Rev 2019; 14 (2): 109-111

DOI: https://doi.org/10.5114/pg.2019.85894

Key words: chronic constipation, aganglionosis, desmosis coli, children.

Address for correspondence: Sylwia Szymańska MD, Department of Pathology, The Children's Memorial Health Institute, Al. Dzieci Polskich 20, Warsaw, Poland, phone: +48 22815 19 60, fax: +48 22815 19 75, e-mail: s.szymanska@ipczd.pl

\begin{abstract}
Introduction: Histopathological diagnosis of chronic constipation in children is difficult and time-consuming because the aetiology of the problem is heterogenous.

Aim: To create the optimal immunohistochemical (IHC) and histological diagnostic protocol using novel antibodies and assessing precisely their patterns.

Material and methods: Twenty-eight paediatric patients were enrolled to the study. The study group consisted of the following: 9 patients with confirmed Hirschsprung's disease (HD), 11 patients with desmosis of the colon (DC) (3) or with chronic constipation of unknown aetiology (3), and eight children operated on due to other problems. Retrospective analysis of full-thickness material from the large intestine was performed. In each specimen the number of ganglion cells was estimated per square millimetre as well as the number of submucosal and intramuscular ganglion cells per ganglion. The following IHC and histological stains were also performed: calretinin, CD117, picrosirius, and trichrome gomori. Patterns (nuclear vs. cytoplasmic vs. membranous) and intensity (strong vs. faint) of the stainings were analysed.

Results: There was no statistically significant difference between groups while comparing the intensity and pattern of each staining, except HD (no staining due to lack of ganglion cells), $p>0.001$. Calretinin was positive in each patient with ganglion cells; however, it did not unequivocally stain all cells identified in routine haematoxylin and eosin staining.

Conclusions: Calretinin is helpful in identifying ganglion cells; however, it cannot replace an experienced paediatric pathologist. In children with chronic constipation it is worth obtaining a full thickness intestinal biopsy in order to perform additional histological and immunohistochemical stains starting with picrosirius red.
\end{abstract}

\section{Introduction}

Motility disorders are a common clinical problem in paediatric patients. The diagnosis is often difficult and time-consuming because its aetiology is heterogenous. Chronic constipation most frequently occurs for functional reasons; however, it may also be caused by alterations in the enteric nervous system. One of the most distinct diseases in newborns is Hirschsprung's disease (HD), caused by failure of neural crest cells to migrate and form normal plexuses and bowel innervations [1]. Other disorders include desmosis of the co-
Ion (DC), neuro-muscular diseases, and inflammatory conditions [2-4]. The treatment depends on aetiology and so it is essential to establish proper diagnosis as soon as possible. The final diagnosis is based on clinical data and histopathology; sometimes imaging tests may be helpful. Rectal biopsy with rapid acetylcholinesterase reaction (frozen sample) is considered as the microscopic gold standard of motility disorder investigation. The evaluation is especially challenging in premature infants because their ganglion cells may be immature and difficult to find, and the ACHE 
reaction may be negative. To make assessment more reliable and quicker, new immunohistochemical (IHC) stains with formalin-fixed paraffin-embedded rectal biopsy markers have been studied. Calretinin is the most highly recommended stain $[5,6]$. It is a calciumbinding protein involved in calcium signalling and is abundantly expressed in neurons. Other markers include the following: microtubule-associated protein-2 (MAP-2), peripherin, neuron-specific enolase (NSE), S100, and CD 117 [7, 8]. If the ganglion cells are present and the child presents clinical symptoms, further diagnosis is very complicated. There are no standards of patient management. Confirmation of desmosis of the colon, a rare condition characterised by total or focal lack of connective tissue net of the circular and longitudinal muscles without any abnormalities within enteric nervous system (ENS), involves full-thickness biopsy and histological staining with Pricrosirius red. Other causes of motility disorders such as neuronal dysplasia or post-inflammatory disorders may require different additional reactions.

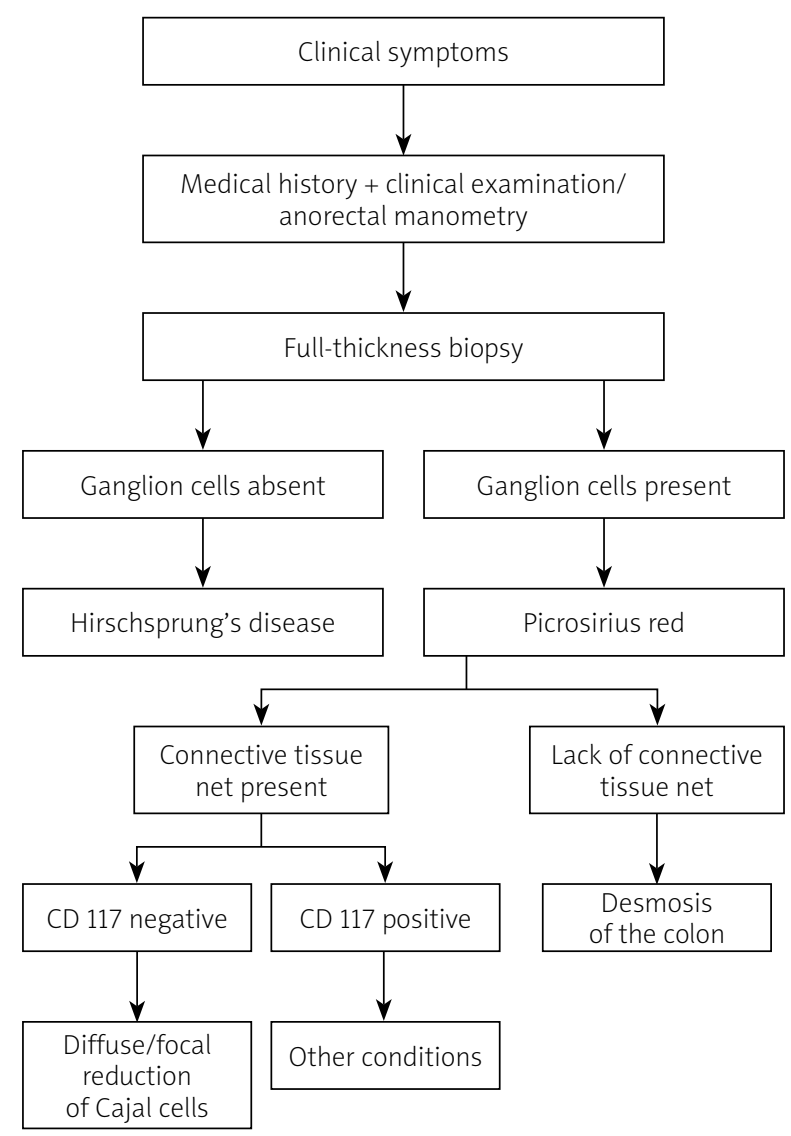

Figure 1. Schedule of diagnostic approach in rectal biopsy in patients with congenital motility disorders

\section{Aim}

The aim of our study was to create the optimal IHC and histological diagnostic protocol using novel antibodies, as well as to assess their patterns precisely.

\section{Material and methods}

Twenty-eight paediatric patients were enrolled to the study. The group consisted of 9 patients with confirmed HD, 11 patients with DC (3) or with chronic constipation of unknown aetiology, and 8 children operated on due to other problems (prior inflammatory bowel disease (IBD). The ages of patients enrolled to the study was from 3 months up to 18 years. They presented with the following: constipation (9); obstruction (6); enterocolitis (1); necrotising ileocolitis (1) and other symptoms of ex-abdominal pain, and diarrhoea (11). Retrospective analysis of full-thickness material from the large intestine was performed. In each specimen the number of ganglion cells was estimated per $\mathrm{mm}^{2}$ counted in 10 HPF (X400) in the muscular plane as well as the myenteric plexus separately. The following IHC and histological stains were also performed: calretinin, CD117, and picrosirius. Patterns (nuclear vs. cytoplasmic vs. membranous) and intensity (strong vs. faint) of the stainings were analysed. The analysis of IHC stains was semi-quantitative as follows: 0 - lack of stain, 1 - faint and focal stain, 2 - strong and focal stain, 3 - strong and diffuse stain.

\section{Statistical analysis}

A descriptive study was carried out for all the variables included in the study. The $\chi^{2}$ was used to compare the categorical values. $P$-value $<0.05$ was accepted as statistically significant.

\section{Results}

Statistical analysis of histopathological changes did not reveal any significant difference between groups, $p>0.001$. Calretinin was positive in each patient with ganglion cells; however, it did not unequivocally stain all cells identified in routine haematoxylin and eosin (HE) staining. Picrosirius red (complete lack of the mesh network of collagen in the intestinal muscular wall) confirmed diagnosis of desmosis coli in each case. Marker CD 117 was not helpful in our group of patients. Therefore, based on our experience and results of statistical analysis, we created a schedule of diagnostic approach in patients with motility disorders (Figure 1).

\section{Discussion}

Motility disorders are a common and difficult clinical problem in paediatric patients. Their diagnosis is often 
time-consuming. Although most of these conditions are functional, in about $5 \%$ of cases, organic disorders can be identified [1] and histopathological examination is necessary. In our care centre there are many children with congenital constipation; therefore, it is mandatory to establish proper diagnosis as soon as possible. Based on our experience and literature review concerning useful immunohistochemical stains in the diagnosis of motility disorders in paediatric patients [2-4], we created a schedule of diagnostic approach in rectal biopsy specimens for this group. Aganglionosis should be excluded firstly because it is the most frequent congenital cause of constipation. We chose calretinin as a marker of mature/immature ganglion cells because it is believed to be the most reliable staining [5, $6,8-13]$. It can be particularly useful for less experienced pathologists. In our study it was helpful because the reaction was positive in all patients with presence of ganglion cells, but it did not stain all cells that were transparent in routine HE reaction. Therefore, we believe that no IHC staining can replace experienced a paediatric pathologist. If $\mathrm{HD}$ is ruled out, full-thickness surgical biopsy of the large intestine should be performed to exclude desmosis of the colon. It is a rare condition, but in our hospital it occurs relatively often. Histological staining with Picrosirius red showing complete lack of the mesh network of collagen in the intestinal muscular wall confirms the diagnosis. In both HD and desmosis of the colon surgery is a treatment of choice, but if they are excluded, we suggest performing c-kit/CD117 staining to prove the presence of Cajal cells, which control bowel muscular activity. Absence or reduction of the number of Cajal cells may cause chronic pseudo-obstruction [6]. In our group we did not identify any patient with this entity. Becheanu et al. [14] suggested that receptors for CD 117 and CD34 are also decreased in Crohn's disease. In our study IBD patients were included as controls, but we did not prove any statistically significant difference between them and the studied group. Further trials on larger numbers of patients are needed. After excluding all these disorders there are still other diseases such as enteric neuropathies or myopathies that should be taken into consideration. It is important to remember that diagnosis of congenital constipation has to be based on the patient's medical history and should also include radiological examination or anorectal manometry. Histopathology is useful in some cases, but in many patients it does not contribute to final diagnosis. Watchful waiting is then the best management because the clinical problem may be functional.

\section{Acknowledgments}

The work was supported by a grant from The Children's Memorial Health Institute for young researchers no. M18/2016, and EU Structural Funds.

\section{Conflict of interest}

The authors declare no conflict of interest.

\section{References}

1. Singer CE, Coşoveanu CS, Ciobanu MO, et al. Hirschprung's disease in different settings - a series of three cases from a tertiary referral center. Rom J Morphol Embryol 2015; 56: 1195-200.

2. Do YS, Myung SJ, Kwak SY, et al. Molecular and cellular characteristics of the colonic pseudo-obstruction in patients with intractable constipation. J Neurogastroenterol Motil 2015; 21: 560-70.

3. den Braber-Ymker M, Heijker S, Lammens M, Nagtegaal ID. Practical and reproducible estimation of myenteric interstitial cells of Cajal in the bowel for diagnostics purposes. Neurogastroenterol Motil 2016; 28: 1261-7.

4. Kidane B, Lam J, Manji F, et al. Histological findings in resected bowel of motility-disordered patients. Am Surg 2015; 81: 187-92.

5. Musa ZA, Qasim BJ, Ghazi HF, Al Shaikhly AW. Saudi diagnostic roles of calretinin in Hirschsprung disease: a comparison to neuron-specific enolase. J Gastroenterol 2017; 23: 60-6.

6. Tran VQ, Truong DQ, Goyens P, Steyaert H. Rectal suction biopsy with calretin in immunohistochemistry in patients suspected with residual aganglionosis after operation for Hirschsprung disease. J Pediatr Surg 2017; 52: 1597-601.

7. Kawai H, Satomi K, Morishita Y, et al. Developmental markers of ganglion cells in the enteric nervous system and their application for evaluation of Hirschsprung disease. Pathol Int 2014; 64: 432-42.

8. Bachmann L, Besendörfer M, Carbon R, et al. Immunohistochemical panel for the diagnosis of Hirschsprung's disease using antibodies to MAP2, calretinin, GLUT1 and S100. Histopathology 2015; 66: 824-35.

9. Gfroerer S, Rolle U. Pediatric motility disorders. Gastroenterology 2015; 21: 9683-87.

10. Chisholm KM, Longacre TA. Utility of peripherin versus MAP-2 and calretinin in the evaluation of Hirschsprung disease. Appl Immunohistochem Mol Morphol 2016; 24: 627-32.

11. Cinel L, Ceyran B, Güçlüer B. Calretinin immunohistochemistry for the diagnosis of Hirschprung disease in rectal biopsies. Pathol Res Pract 2015; 211: 50-4.

12. Małdyk J, Rybczyńska J, Piotrowski D, Kozielski R. Evaluation of calretinin immunohistochemistry as an additional tool in confirming the diagnosis of Hirschsprung disease. Pol J Pathol 2014; 65: 34-9.

13. Najjar S, Ahn S, Kasago I, et al. Image processing and analysis of mucosal calretinin staining to define the transition zone in Hirschsprung disease: a pilot study. Eur J Pediatr Surg 2019; 29: 179-87.

14. Becheanu G, Manuc M, Dumbravă M, et al. The evaluation of interstitial Cajal cells distribution in non-tumoral colon disorders. Rom J Morphol Embryol 2008; 49: 351-5.

Received: 29.10 .2018

Accepted: 19.11.2018. 